\title{
DFT based Channel Estimation in OFDM System
}

\author{
R. Prakash Kumar \\ Department of ECE, CVR College of Engineering, Hyderabad, India. \\ Email:Prakash.rachmagdu@gmail.com
}

\begin{abstract}
Multi carrier modulation is Orthogonal Frequency Division Multiplexing (OFDM), dividing the large band spectrum into small (narrow) band spectrum. OFDM offers flexibility in adaptation to time varying channel condition by adopting the parameters at each subcarrier accurately. Due to the multipath effect the OFDM system suffers Inter Symbol Interference (ISI).ISI can be reduced by introducing the guard band (cyclic prefix).Time-varying channel estimation is an important activity in next generation wireless communication. Channel estimation is required mainly for the significant signal processing operation in multiple input multiple output MIMO- OFDM systems. In this project DFT - based channel estimation based on block type arrangement is implemented in MAT Lab. The bit error rate (BER) of DFT (Discrete Fourier Transform)based channel estimation is less than the BER of LS (Least Square) and MMSE (Minimum Mean Square Error) based channel estimation with same signal to noise ratio (SNR).
\end{abstract}

Index terms: OFDM, channel estimation, MMSE and LS.

\section{INTRODUCTION}

During the past few years, there has been an explosion in wireless technology. This growth has opened a new dimension to future wireless communications whose ultimate goal is to provide universal personal and multimedia communication without regard to mobility or location with high data rates. To achieve these high data rates, the next generation during the past few years, there has been an explosion in wireless technology. This growth has opened a new dimension to future wireless communications whose ultimate goal is to provide universal personal and multimedia communication without regard to mobility or location with high data rate[3]s. To achieve these high data rates, the voice high quality, data, picture and video transmission should support in the next generation personal communication [2]. To transmit these services require data rate in terms of Mega bits per seconds (Mbps) Whenever data is transmitted high data rates, the response of the channel is spread over the many symbol periods, which leads to inter symbol interference (ISI)[4][10].To reduce the ISI usual technique is OFDM.In this technique the entire bandwidth is divided into many narrowband sub channels which are synchronous in transmission. All the sub channels or subcarriers are orthogonal to each other to eliminate the effect of delay spread. To reduce the co-channel interference in mobile environment and dispersive -fading limitations by adding turbo codes to OFDM system [4][5].

\section{BLOCK DIAGRAM OF DFT BASED OFDM SYSTEM}

The block diagram of the proposed system is shown in Fig1. At the receiver we are estimating the transmitted signal by adding the DFT block.

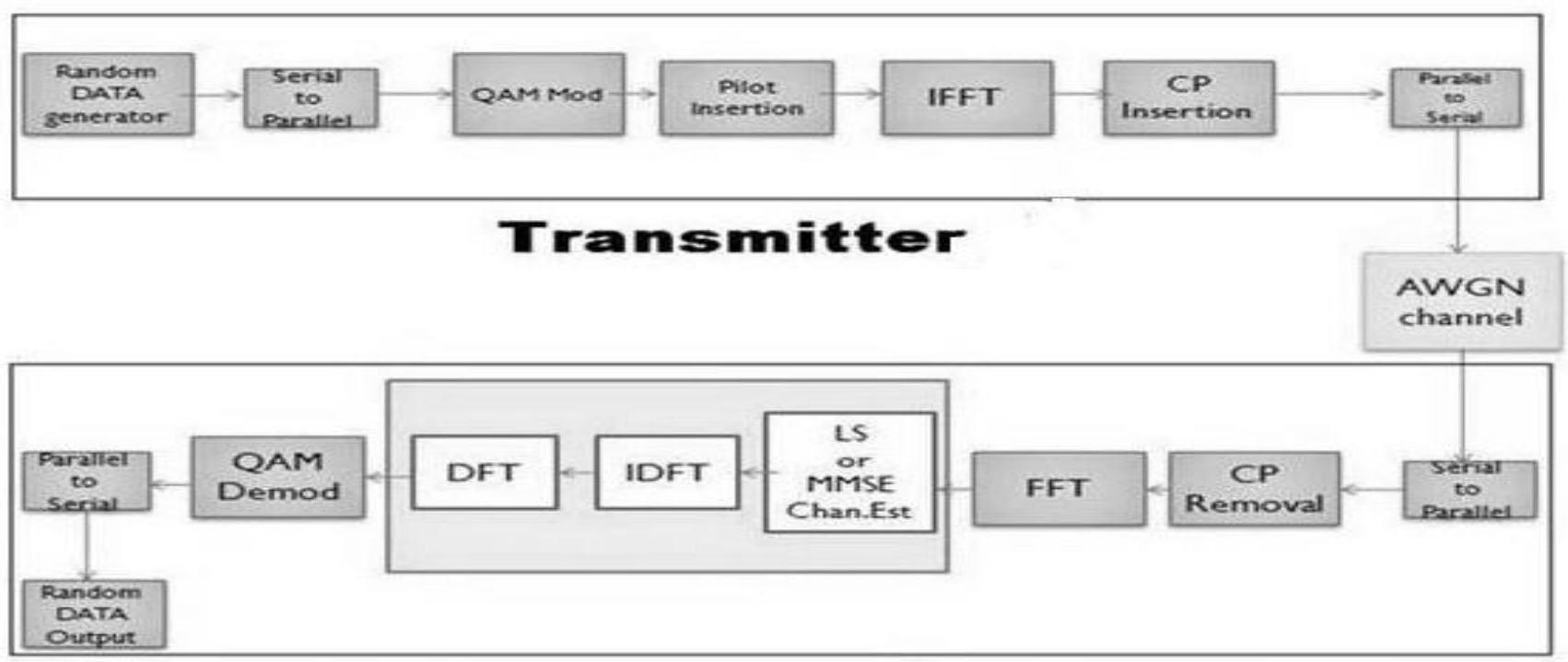

Receiver

Fig1.Block diagram of DFT based OFDM system 


\section{DFT BaSed Channel Estimation PerformanCe OF MSE}

In the MIMO-OFDM context with $\mathrm{Nt} \leq(\mathrm{M} / \mathrm{CP})$ transmitter antennas and $\mathrm{Nr}$ receiver antennas, the (MSE) on the $\mathrm{k}$-th subcarrier is equal to:

$$
\operatorname{MSE}(k)=\frac{\sum_{i=0}^{N_{t}} \quad \sum_{i=0}^{N_{r}} E\left[\|\widehat{H(k)}-H(k)\|^{2}\right]}{N_{t} N_{r}}
$$

Where $\mathrm{H}(\mathrm{k})$ and $\mathrm{H}(\mathrm{k})$ represent the estimated frequency channel response and the ideal one respectively.

For time and frequency selective MIMO ,MSE performance provided with FFT $2 \times 2$ MIMO system and its size is 1024.null symbol insertion is used for providing the Orthogonality between the subcarriers, for this subcarriers MSE is same and there is no border effect. By the impulse response from the first $\mathrm{CP}$ samples obtained the total power of the channel [7].

The performance of MSE and channel power is degraded due to null subcarrier are inserted on the edge of the spectrum [6], but number of subcarriers are increases the broader effect is observable.

LS and MMSE estimation is based on pilot frequency for comb-type.LS performance is not better than the LMS or MMSE complexity is more in MMSE. This will reduced by deriving the singular value decomposition with optimal low rank estimation [3][5]. Comb-type estimation depends on interpolation type such as second order, linear, spline. And low pass. Out of all these interpolations techniques second order interpolation has better performance than linear interpolation [3]. Low BER is achieved by time domain interpolation to linear interpolation [9].

\section{ALgORITHM For DFT-BASED CHANNEL ESTIMATION}

The channel gain of the $\mathrm{k}^{\text {th }}$ subcarrier obtained from the LS channel estimation is denoted by $\mathrm{H}(\mathrm{k})^{\wedge}$ taking IDFT of the LS estimation is

$$
\{H[k]\} \begin{aligned}
& N-1 \\
& k=0
\end{aligned}
$$

$$
\begin{aligned}
\operatorname{IDFT}\{\widehat{H}[k]\}= & h[n]+z[n] \\
& \triangleq \widehat{h}[n],
\end{aligned}
$$

Where $Z[n]$ denotes the noise component in the time domain. Ignoring the coefficients $h^{\wedge}[n]$ that contain the noise only, we can define the coefficients for the maximum channel delay $\mathrm{L}$ as

$$
\widehat{h}_{D F T}[n]=\left\{\begin{array}{cc}
h[n]+z[n], n=0,1,2 \ldots L-1 \\
0, & \text { otherwise }
\end{array}\right.
$$

And transform the remaining $\mathrm{L}$ elements back to the frequency domain as follows
The Algorithm of the performed task is given below. Transmitter:

Step.1: Encode the message data by coding. In this paper we are using the Convolution coding.

Step.2: QAM is used for mapping of encoded data.

Step.3: for synchronize and estimating the channel some known bits are inserted to the modulated data called pilot symbols.

Step.4: Convert the time domain signal into the frequency domain by IFFT of step 3 data.

Step.5: To avoid the Inter symbol interference, insert the guard period between the output data of step. 4 .

Step.6: Transmit output data through the AWGN channel. Receiver:

Step.7: received data having the noise, removed by using the filters. Guard period is removed and compute the FFT (i.e frequency domain into the time domain signal.)

Step.8: Channel estimate the filter co-effients[4] for received signal and other known information (such as modulation type and cyclic prefix length etc) apply the different kinds of estimating technique like LS, MMSE and DFT.

Step.9: Remove the channel effect from the output of step.7 with the help of the output found in step.8.

Step.10: Pilot removal, demodulation and decoding operation performed for the step 9 data.

Step.11: Calculate the Bit Error Rate (BER) for the step 10 data with channel estimation technique.

\section{SimULATION RESULTS}

In this paper comb type pilot insertion scheme is implemented. We have discussed and simulated 3 types of channel estimation techniques[3][8]. Those are; LS-linear (Least Square Channel Estimation with Linear Interpolation), LS-Spline (Least Square Channel Estimation with Spline Interpolation) and MMSE (Minimum Mean Square Error) method [2].

These techniques are not that much efficient when compared to DFT based channel estimation. The Bit error rate (BER) and MSE (Mean Square Error) are improved in DFT- based channel estimation.

We have simulated the above mentioned techniques (LS-linear, LS-Spline and MMSE) in both scenarios. One with DFT- based Channel Estimation and another without DFT-based channel Estimation. In every method, the results of the method are compared with the true channel and MSE is calculated[10]. The number of symbol errors also calculated.

The Simulation Results are given below, Case (i): $\mathrm{SNR}=10 \mathrm{~dB}$

$$
\widehat{H}_{D F T}[k]=\operatorname{DFT}\left\{\hat{h}_{D F T}[n]\right\}
$$



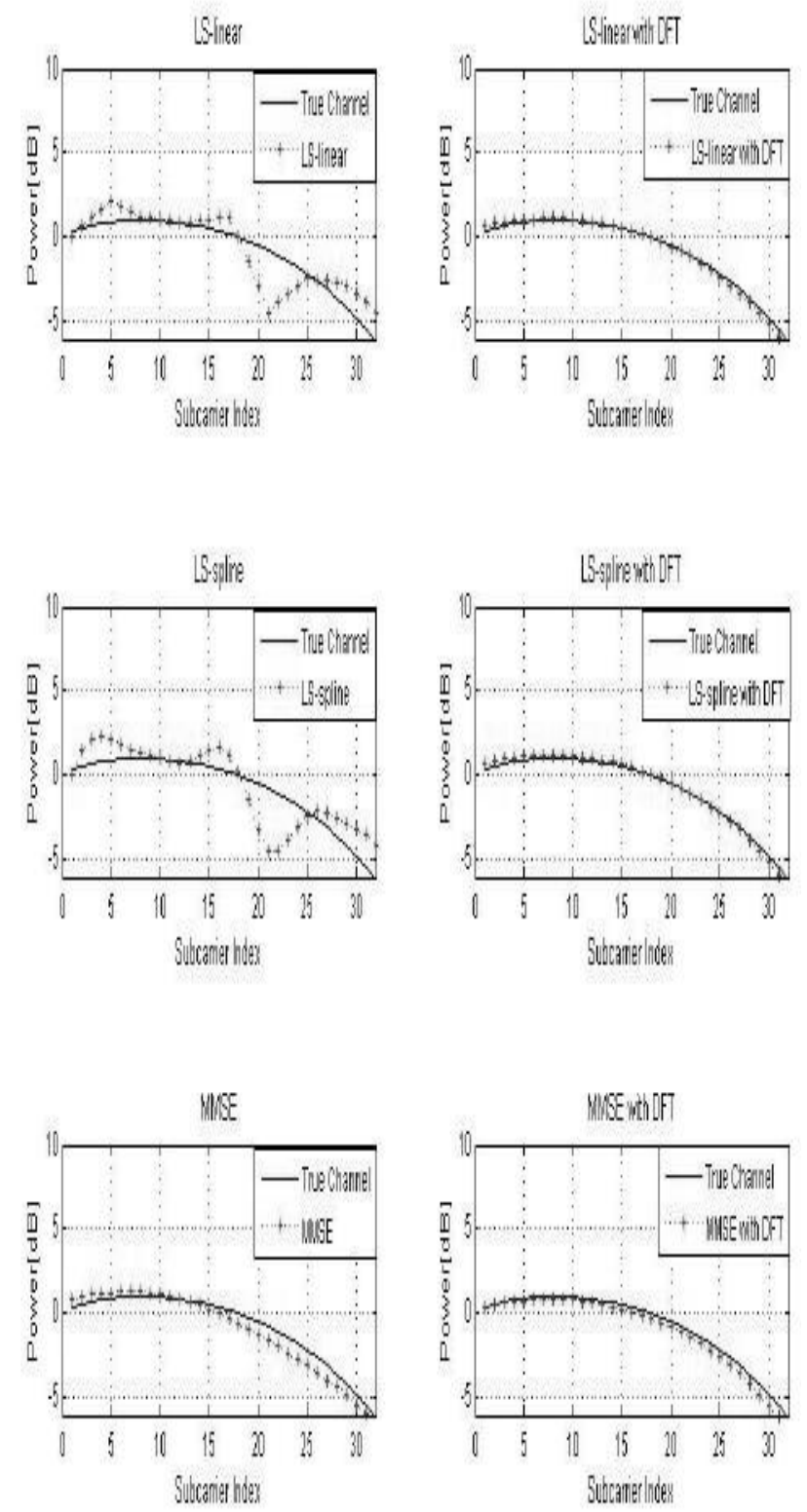

Fig.2: The Channel estimation of different methods with and without DFT based estimation for $\mathrm{SNR}=10 \mathrm{~dB}$

Fig.2 shows that Interpolation should be done to estimate the data. Interpolation can be done in two ways, one among them is Linear and the other one is spline interpolation[5]. In the Linear method, the samples are connected by using linear algebraic equation whereas in the Spline Interpolation the samples values are connected by using polynomial equations. For the lower SNR values(10dB in this case), Linear and Spline Interpolation techniques fail to estimate the exact data[7].
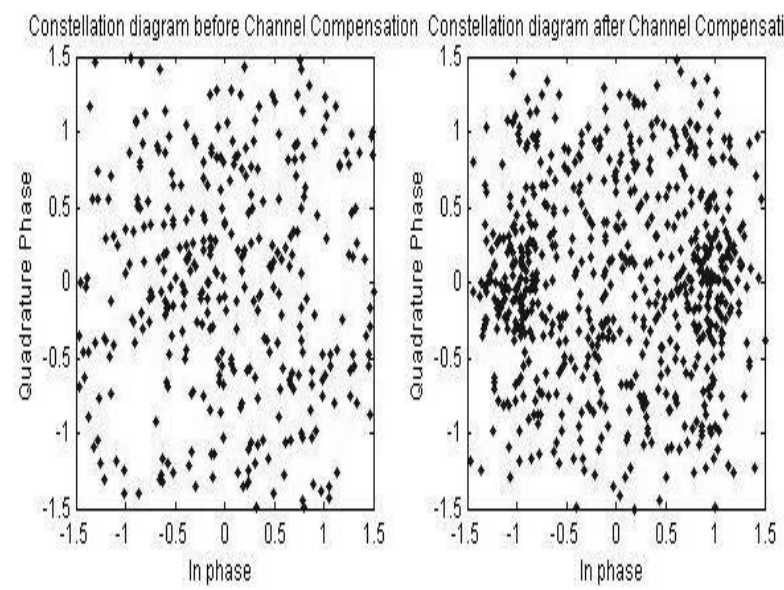

Fig.3: Comparison of Constellation diagrams before and after channel compensation for $\mathrm{SNR}=10 \mathrm{~dB}$

Constellation of QAM modulator is effected with AWGN (additive white Gaussian noise) component. Here we are comparing Constellation diagrams of QAM modulator before and after the channel compensation for $\mathrm{SNR}=10 \mathrm{~dB}$ is shown in Fig. 4 .

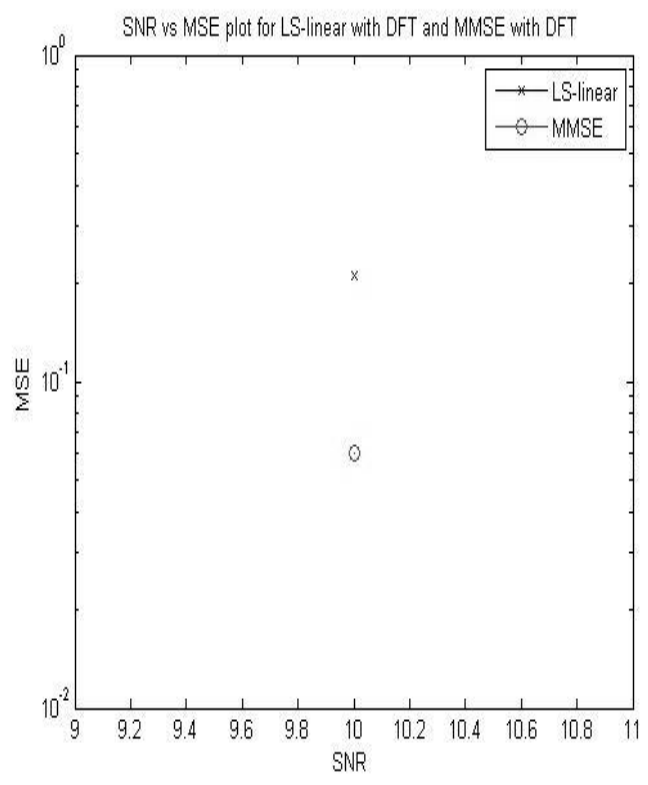

Fig.4: The Plot of SNR vs. MSE for SNR=10dB

SNR vs MSE graph is plotted for $\mathrm{SNR}=10 \mathrm{~dB}$. We can observe that the MSE for MMSE is less than the MSE of LS method.

Number of symbol errors $=1602$

MSE of LS-linear $=2.1246 \mathrm{e}-001=0.21246$

MSE of LS-spline $=2.6341 \mathrm{e}-001=0.26341$

MSE of MMSE Channel Estimation $=6.0107 \mathrm{e}-002=$ 0.060107

MSE of LS-linear Channel Estimation with DFT=3.6950e$002=0.036950$

MSE of LS-spline Channel Estimation with DFT=3.7424e002 $=0.037424 \mathrm{MSE}$ of MMSE Channel Estimation with $\mathrm{DFT}=3.5421 \mathrm{e}-002=0.035421$.

Case(ii) :SNR=30dB. 

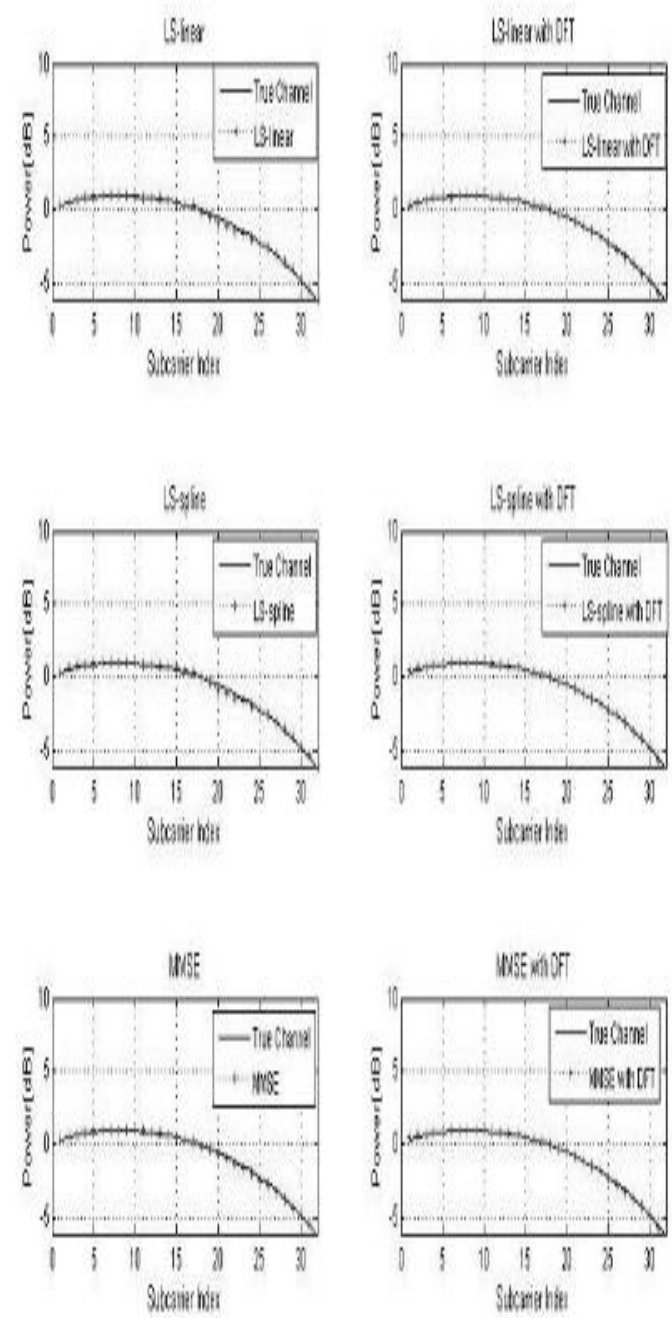

Fig.5: The Channel estimation of different methods with and without DFT based estimation for $\mathrm{SNR}=10 \mathrm{~dB}$
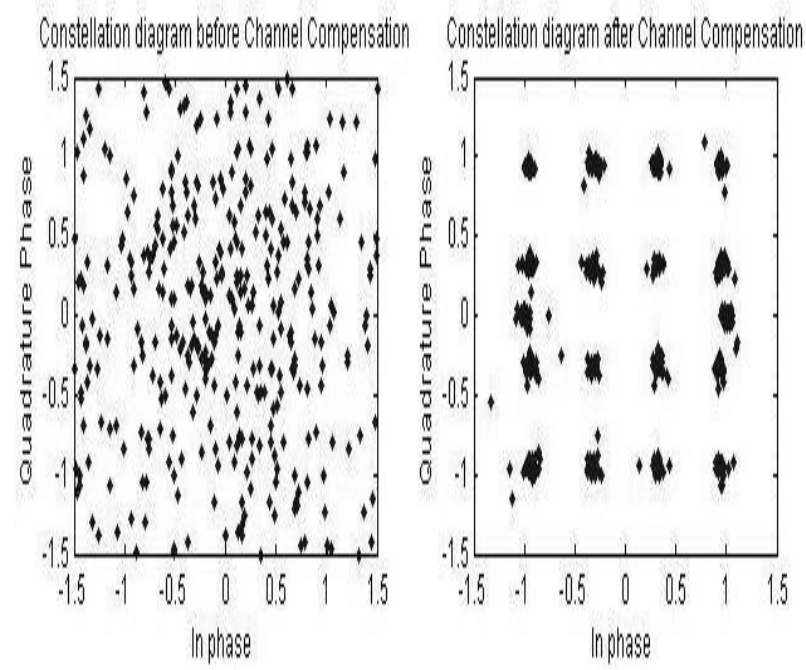

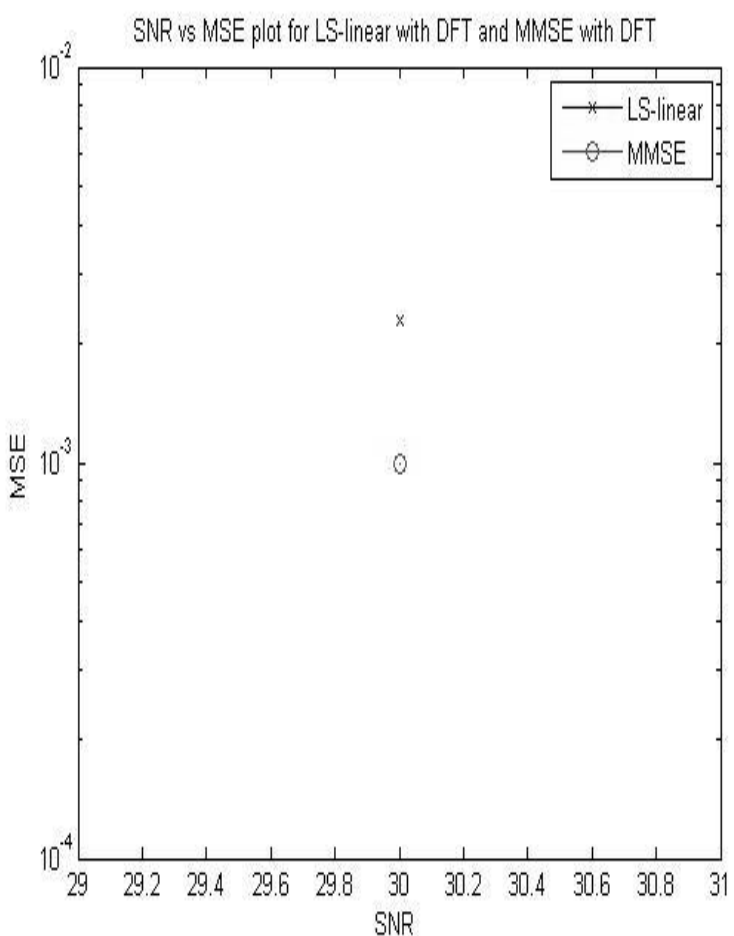

Fig.7 Plot of SNR vs. MSE for SNR=30dB

Number_of_symbol_errors $=14$

MSE of LS-linear $=2.2877 \mathrm{e}-003=0.0022877$

MSE of LS-spline $=2.7134 \mathrm{e}-003=0.0027134$

MSE of MMSE Channel Estimation $=9.9295 \mathrm{e}-004=$ 0.00099295

MSE of LS-linear Channel Estimation with DFT= $4.0627 \mathrm{e}-004=0.00040627$

MSE of LS-spline Channel Estimation with DFT=3.7935e$004=0.00037935$

MSE of MMSE Channel Estimation with DFT $=3.6774 \mathrm{e}-$ $004=0.00036774$.

\section{CONCLUSIONS}

Wireless communication transmits with high data rate and reliable from source to destination, this is achieved by OFDM technique. Major problem in OFDM system is fading effect[7][10]; this can be compensated at the receiver by channel estimation techniques. In this paper we are proposing the DFT channel estimation is better performance than the MMSE and LS techniques. The simulation is done for SNR of $30 \mathrm{~dB}$. The MSE for DFT based system $\mathrm{dB}$ is 0.00036774 and $\mathrm{LS}$ estimation is 0.00037935 . The advantage of DFT based technique it does not require any prior channel information.

Fig.6: Comparison of Constellation diagrams before and after channel compensation for $\mathrm{SNR}=30 \mathrm{~dB}$ 


\section{REFERENCES}

[1] M. Sandell Edfors and J.-J. Van, "OFDM Channel Estimation By Singular Value Decomposition", IEEE Trans. Communication, vol. 46, no. 7, (1998) July, pp. 931-39.

[2] J.-J. van de Beek, O. Edfors, M. Sandell, S. K. Wilson and P. O. Börjesson, "On Channel Estimation in OFDM Systems", IEEE 45th Vehicular Technology Conference, Chicago, vol. 2, (1995) July, pp. 815-819.

[3] Y. Zhao and A. Huang, "A Novel Channel Estimation Method for OFDM Mobile Communication Systems Based on Pilot Signals and Transform-Domain Processing", Vehicular Technology Conference, Phoenix, AZ, (1997) May, pp. 2089-93.

[4] A. Dowler, A. Doufexi, and A. Nix, "Performance Evaluation of Channel Estimation Techniques for a Mobile Fourth Generation Wide Area OFDM System", IEEE 56th Vehicular Technology Conference, Canada, vol. 4, (2002) September, pp. 2036-40.

[5] H. Minn and V. K. Bhargava, "An Investigation into TimeDomain Approach for OFDM Channel Estimation", IEEE Transactions on, Broadcast, vol. 46, no. 4, (2000) December, pp. 240-48.
[6] Y. Kang, K. Kim and H. Park, "Efficient DFT-based channel estimation for OFDM systems on multipath channels", IET. Communications, vol. 1, no. 2, (2007) April, pp. 197-202.

[7] Y. Lee, H. Shin, and H. Kim, "Channel Estimation Based on a Time-Domain Threshold for OFDM Systems", IEEE Transactions on, Broadcast, vol. 55, no. 3, (2009) September, pp. 656-663.

[8] J.-J. Van De Beek, O. Edfors, M. Sandell, S. K. Wilson, and P. O. Borjesson, "OFDM Channel Estimation by Singular Value Decomposition", IEEE Trans. on Communications, vol. 46, no.7, (1998) July, pp. 931-939.

[9] Peter Hammarberg, Ove Edfors, "A comparison of DFT and SVD based channel estimation in MIMO OFDM systems", The 17th Annual IEEE International Symposium on Personal, Indoor and Mobile Radio Communications (PIMRC'06), 2006.

[10] M.Belotserkovsky, "An equalizer initialization algorithm for OFDM receivers," Digest of Technical Papers International Conference on Consumer Electronics, 2002, pages 372-373, 20. 